\title{
RELATIONAL THINKING IN EDUCATION: TOPOLOGY, SOCIOMATERIAL STUDIES, AND FIGURES
}

\author{
MATHIAS DeCuYPERE* \& MAARTEN Simons
}

Laboratory for Education and Society, University of Leuven, Leuven, Belgium

\begin{abstract}
Over the last few years, different sociomaterial research orientations have emerged. In this article, we argue that most of these orientations are relying on a relational mode of thinking, that is, a way of conceiving of educational practices in terms of the relations between the different actors present in these particular practices. In doing so, these various sociomaterial studies share many of their theoretical assumptions with social topology, an approach inspired by the mathematical field of topology. In educational research, however, this connection between sociomaterial and sociotopological accounts is not commonly made. Therefore, this article calls for a more intricate interweaving of topological thinking with better-known sociomaterial approaches. Furthermore, we assert that using visualisations might play a crucial role in this respect. To that effect, we introduce the Foucauldian and Deleuzian notion of the diagram. This notion of the diagram, as the technique that brings the orders of the visual and the articulable together, is conceived as a promising technique in order to investigate different aspects of educational practices. In a concluding section, the article offers some suggestions as to what the general potential of adopting such relational studies in the field of education might be.
\end{abstract}

To cite this article: Mathias Decuypere \& Maarten Simons (2016): Relational thinking in education: topology, sociomaterial studies, and figures, Pedagogy, Culture \& Society

To link to this article: $h$ ttp://dx.doi.org/10.1080/14681366.2016.1166150

*mathias.decuypere@kuleuven.be 


\section{Introduction}

Over the last few years, different research orientations aiming to investigate both the social and the material dimensions of daily educational practices have emerged. Advocates of these orientations argue that material dimensions of educational practices are all too often ignored since much conventional educational research tends to place the traditional human subject in educational practices (students, teachers, directors, ministers, ...) centre-stage. Thus, the argument goes, a whole realm of equally important material agents is not being accounted for (Fenwick, Edwards, and Sawchuk 2011). In trying to equally account for this material realm, educational researchers (e.g., Fenwick and Landri 2012; Landri and Neumann 2014) are gradually showing more interest in, and adopt, these relatively recent sociomaterial approaches. Being relatively new, a conceptual common ground is emerging but has not yet been found: some studies in the field of education typify themselves as being actor-network theory (ANT) studies (e.g., Fox 2005; Habib and Wittek 2007), others as being assemblage studies (e.g., Gorur 2011; Koyama and Varenne 2012), still others as socio-technical or sociomaterial studies (e.g., Luck 2008; Orlikowski 2007), and so on.

It is, however, not the aim of this article to try to bring conceptual unity to these nomenclatures. Instead, we will show that what these approaches have in common is that they are keenly interested in materiality as well as in relations. This emphasis on relations is similarly adopted by social topology (ST), an approach inspired by the mathematical field of topology. Even though this common relational focus has been highlighted by some (e.g., Law 2002; Mol and Law 1994), this topological approach is relatively infrequently adopted in sociomaterial studies (and this especially applies to educational studies). Therefore, this article calls for a more intricate interweaving of topological thinking with more well-known conceptual frameworks of sociomaterial studies (e.g., ANT). Such interweaving, we argue, could provide a fruitful vantage point for rigorously understanding and investigating the relationality within educational practices. Furthermore we assert that using visualisations might play a crucial role in this respect.

The structure of this article is as follows. The first section elaborates the general argument that relational thinking constitutes a common ground of both sociomaterial and topological studies. We start by elucidating this relational thinking and illustrate what a stringent application of such thinking entails. Thereafter we introduce some central tenets of (social) topology. We argue that topology constitutes a fruitful approach to applying such relational thinking. Furthermore, we illustrate this argument by some examples of educational studies that have already been conducted in this sociotopological vein. This will bring us to the conclusion of this first section, pointing to a distinction that both sociomaterial and sociotopological studies of education need to attend to, namely that there is a profound difference between stating that educational practices are relationally constituted and showing how precisely this looks like. The second section proposes some reflections on how to conduct research in a relational vein, in such a way that one shows the relational distribution (the form) of educational practices. For that purpose it will be argued that topological conceptions of forms and figures, and concomitant visualisations, are especially instructive with respect to the presentation and analysis of such distributions. Furthermore, we propose some outlines as to how to conceive of such visualisations, especially in relation to both the (aspects of the) realities these figures attempt to present and the text in which they are embedded, and what such visualisations enable people to see and analyse. A third and concluding section offers some suggestions as to what the general potential of adopting relational studies in the field of education might be, namely the possibility of searching for a mode of existence that is typical for the particular educational practices under investigation. We draw this article to a close by arguing that there are specific ways for various educational practices to exist and that relational studies might have a focal role in opening up a space to consider and specify 
what precisely makes these modes of existence characterizable as being educational modes of existence.

To start with, however, we wish to make three disclaimers. First, despite topology's mathematical origin, we largely focus on the branch of applied topology that is known as social topology, since it uses topological reasoning to provide new ways of looking at, and interpreting, social practices. Second, this article uses the umbrella term sociomaterial studies in order to characterise the many studies in the field of education that give an account of the intricacies and tight interwovenness of the social and the material in educational practices. Hence, in this article, the term sociomaterial studies includes ANT studies, assemblage studies, some branches of educational practice studies, etc. Related to this, and the final disclaimer, is that this article should not be conceived as being a comprehensive review of the sociomaterial and sociotopological literature in the educational field. Rather, it constitutes a theoretical and methodological argument for a rigorous application of relational thinking and offers some propositions in order to effectuate research in this vein.

\section{Relational thinking}

Traditionally, social theories are primarily interested in the human dimensions of the settings they are investigating and scrutinise these dimensions to a far greater extent than the material dimensions of these settings. Moreover, the majority of studies that do take materiality into account do this largely by including material objects as effectual elements. Material or technological components are then considered important in as far as they enable or disable certain human actions or in as far as they facilitate or constrain the effectuation of certain tasks performed by a human individual. Hence, in the larger part of contemporary social theories, the inclusion of materiality most of the time amounts to an instrumentality vis-à-vis the conduct of human tasks (e.g., Callon and Muniesa 2005; Fenwick, Edwards, and Sawchuk 2011; Schatzki 2010; Waltz 2006). Sociomaterial studies, on the other hand, jointly focus on both the social and the material dimensions of different educational settings in view of researching the active role that both these components play. Often, these sociomaterial studies operate in a relational framework. This relational point of view not only includes both social and material aspects in analysing how a practice under investigation is constituted precisely, but equally, and more importantly, states that it is the relations between these different actors that are crucial. In other words, rather than focusing on social and material actors as being distinct entities, relational thinking places the primacy on the prevalent relations in a setting. In doing so, it asserts that in order to understand practices, it is important to look at the relations between different actors - and this to such an extent that actors are, in fact, the result of the relations they uphold with other actors.

In order to elaborate this argument, relational thinkers often make a distinction between the settings where certain activities take place on the one hand and the specificity of these settings on the other (e.g., Latour 2013; Schatzki 2010). The term setting then denotes an arrangement of interconnected (social and material) entities that points to where, for instance, education takes place. This 'where' might be confined to a classroom (as a physical arrangement of teacher, pupils, desks, computers, and so on), but it might equally point to a non-physical arrangement such as the broader realm in which this classroom is situated (as an arrangement of education policy, district welfare, and so on). Relational thinking conceives of both such physical and non-physical arrangements as consisting of relations between actors that give shape to what is being done and what can be done. In other words, there is no need for context here anymore, that is, if context is used in an overarching (framing) and/or clarifying sense. Instead, rather than being considered as an overarching factor to explain or understand practices, in a relational view 'context' constitutes a broader set of relations between very 
specific actors as well. That is to say, 'context is an outcome of activity or is itself a set of practices' (Edwards 2009, 3). Rather than being static or structuring, in relational thinking so-called context should equally be approached as a specific enacted result of various relations. The district welfare or education policy of a region, for instance, is then not conceived as a structuring given in which a classroom is situated, but rather as a describable assemblage whose relations enable and/or prevent particular actions. 'Education policy' is then no big monolith but a specific arrangement of documents, tables, websites, ministers, cabinets, and so on, that relationally gives shape to what can or cannot happen in a classroom setting. In that sense, the type of relations is of importance: the relation between a policy document and a teacher, for instance, might be a relation of instruction, but equally a relation of modification or neglect.

Eventually, the relations that are formed between social and material actors in such settings give shape to different practices. Practices relate to settings in the sense that they point to the specific spatiotemporal features that emerge in these settings and that are composed by the relations between both social and material actors. As such, practices not only pertain to settings, but equally point to the specificity of the actors in these settings and to the doings, sayings, activities, understandings and routines that take place in such settings. A teaching practice, for instance, is situated both in a classroom setting and an education policy setting, but - as a teaching practice - points to the specificity of this teaching, rather than (only) to the arrangements in which it is situated. That is to say, in a relational vein, 'teaching' is not only the result of a web of relations between different actors but equally enacts particular effects that can only be discerned by considering the types of relations that are involved in a certain setting. In that sense, the focus of relational thinking is not only directed to the 'what' or 'who' (what/who is positioned in a particular setting), but also to the how and the where: What do these relations look like? What is specific about these relations? Where are these to be found? Are there actors that are almost never related to? Are there actors with which many actors nearly always relate? A teaching practice, for instance, might turn out to be specific in as far as there are not only pupil-teacher relations established, but equally that a certain object (a rock, a newspaper article, ...) is being related to by all humans in the classroom setting. This may be something that attracts attention as far as particular sorts of time and space are enacted. How the sociomaterial actors present in a particular setting shape a teaching practice is then further exemplified by asking questions such as: What do the ways of doing things in a classroom together look like? Where is it that teaching occurs? Which sorts of time and space are enacted likewise? (Landri 2012; Mol 2000; Schatzki 2010).

In posing such questions, relational thinking discards traditional distinctions between agency and structure. Just as agency is never the sole feat of one singular actor, what is conventionally designated as structuring factors (e.g., order, stability) is equally enacted relationally: the 'structure' of a practice is not considered as being there a priori, but rather comes into being as a consequence of the relations between different actors (Latour 2005; Nespor 2004). In that sense, structures (but also actors) are not pre-givens, but rather specific enactments of constellations of relations in a certain setting, constellations that cause something to come into being as, for instance, stable and ordered. The strong consequence, then, is that such constellations need to be described empirically, instead of assumed a priori.

In sum, relational thinking is centrally concerned with settings in which actors relate with each other and in which, as a result, a specific way of doing things - a practice - emerges that is constantly in the making (instead of pre-given) and that can only be unfolded by means of empirical investigation (Fenwick and Edwards 2010; Law 2009). The question then arises how to put these relational tenets to work, that is, how to investigate practices with a relational disposition as point of departure. As stated in the introduction, most educational studies answer this question largely by adopting a sociomaterial 
framework. However, in the following section we argue that social topology, with its focus on figures and forms, equally constitutes a promising approach in this respect.

\section{1. (Social) topology}

In its mathematical origin and in a technical sense, topology is a subbranch of geometry. Both domains are generally concerned with space and with the properties of objects in that space, although they approach this notion of space in a radically different way:

Geometry (geo-metry) has to do with measurement, while topology disregards measurement or scale, and deals only with the structure of space qua space (topos) and with the essential shapes or structure of figures. If one deforms a given figure continuously, (i.e., does not separate points previously connected and, conversely, does not connect points previously separated), the resulting figure is considered the same. (Plotnisky 2003, 99)

Hence, generally spoken, mathematical topology does not operate within a framework of a fixed set of global (Cartesian) coordinates, which enable us to measure a certain space, or of (Newtonian) dynamics, which enable us to describe how objects move in space. Instead of that, topology is concerned with space qua space. Studying space qua space signifies not approaching space as if it were contained within an extensive box of underlying transcendent Cartesian coordinates, but rather as being defined exclusively through the intensive features of the spatiality of the figures: rather than considering the size of a figure by means of a system of coordinates, what is important in topology is how the relationships between various points/agents enact a space themselves. Thus, topologists do not conceive of space as being there already. Rather, space is shaped by its very contents (De Landa 2002; Martin and Secor 2014). As far as the topological study of figures is concerned, then, the focus lies upon continuity of shapes, as the above quotation illustrates. It is thus that in topology a circle is equivalent to a triangle, or a mug to a donut: without breaking the original figure (that is, keeping the relations between the various points of the figure), one can deform a circle or a mug in such a way that one ends up with another shape (a triangle or a donut respectively). In that sense, the original (circle, mug) and the eventual (triangle, donut) figure are called homeomorphic: Even though a circle needs squeezing in order to be rendered as a triangle, in topological thinking the circle and the triangle are considered equivalent since they hold a similar relational form (Hinchliffe et al. 2013; Law 2002). In other words, topology focuses on whether and how the form of a figure (that is, the relations between its different points) is retained if it is bent, squeezed, etc., and whether and how shapes that in the end look completely different relate towards each other.

How and why has this mathematical domain found its way into the social sciences? The potential of topology in the social sciences is mostly made manifest in terms of its potential to think differently about practices under investigation, how these practices are to be studied, and how we conceive of spatial and temporal features in and of such practices. Social topology is thus often framed as an alternative to more traditional spatial and temporal conceptions in social sciences that conceive of space and time as a priori givens. Furthermore, the adoption of topology in the social sciences is not particularly focused on mathematical formulae. Instead, most of the sociotopological field is postmathematical in the sense that, primarily, a topological way of looking at and conceiving of the specific practices under investigation is being adopted (Martin and Secor 2014 - but see Sha 2012). In this respect, relationality (as outlined in the previous section) is the prime analytic lens: social topology studies stress the importance of empirically describing the emerging relations between actors over the absolute size or shape of these actors (e.g., Hinchliffe et al. 2013; Marres 2012; Thompson and Cook 2015). The term 'actor' must be understood very broadly and can be anything, so long as it has an 
active role - which is similar to sociomaterial studies: it might be a vessel, a pump, a disease, a human being, etc. Michel Serres has made this relational point particularly clear by making an analogy with a ball game (rugby):

Configurations or fixed places are important when the players don't move - just before the game begins, or when certain established positions are called for at various points in the game - scrimmages or line-outs. They begin to fluctuate as soon as the game begins, and the multiple and fluctuating ways of passing the ball are traced out. (...) The ball is played, and the teams place themselves in relation to it, not vice versa. As a quasi object, the ball is the true subject of the game. It is like a tracker of the relations in the fluctuating collectivity around it. The same analysis is valid for the individual: (...) the skilled player knows that the ball plays with him or plays off him, in such a way that he gravitates around it and fluidly follows the positions it takes, but especially the relations that it spawns. (Serres and Latour 1995, 108)

Serres, an advocate of the introduction of topology into the social sciences, is stressing the importance of relations (passing and movements) here, over and above the actors (the ball, the players) populating a particular setting (the pitch). How do actors place themselves in relation to this 'tracker of relations'? Where do they do this? What does this fluctuating collectivity look like? In trying to answer such questions, social topology heavily draws on topology's concept of forms in general and the idea of homeomorphism in particular. That is to say, a central interest of a wide range of ST studies has precisely been how the form of particular settings is being enacted relationally. Analogous to the example of the cup and the donut, ST contends that if two settings retain a same (that is, a continuous) form, they can be conceived as being homeomorphic, transforming themselves from one arrangement into another without discontinuity (Mol and Law 1994, 664). This is equally reminiscent of sociomaterial studies which also aim to scrutinise the relational distribution of a particular setting, although these studies traditionally do not place particular emphasis on this notion of form (they usually speak about compositions instead - Latour 2005). Hence, the general argument that settings are to be studied by means of relations in order to scrutinise the particular form of a practice is given more emphasis in social topology. Drawing again on Serres' analogy of the ball game, it is precisely these relations that permit us to scrutinise the specific ways of doing things together: Which sorts of (relational) acting and doing are effectuated? Which sorts of space and time are enacted? Such questions are not primarily directed at who passes at whom, but rather at how any player is positioned in relation to any other player, how this positioning changes over time and how, as a result, different sorts of time (play time and injury time, for instance) and space (space in terms of lines of attack and defence, for instance) come relationally into being. That is to say, according to social topology, relations between actors in a particular setting equally enact particular sorts of space and time, which eventually enables us to say something about the specificity of the setting of a ball game practice (Lash 2012; Lury, Parisi, and Terranova 2012).

Drawing inspiration from the mathematical field of topology, a sociotopological understanding of space and time differs from linear (chronological) and metric (Cartesian) conceptions that are traditionally deployed in the larger part of the social sciences. In topological terms, time is relational. This implies, for example, that traditional 'singular' objects can be considered as being multitemporal, being the manifestation of a network in which different temporal elements are gathered together: the invention of the diesel engine, patents, new electronic technologies, all come together in one single object that we conventionally call 'car' (Serres 1979; Serres and Latour 1995). A similar relational approach is made towards space. Law (2002), for instance, has elaborated upon notions such as regional, network, and fluid spaces, all enacted through the relations between different sociomaterial actors. Depending on the particular distribution of these relations, the same constellations of actors enact different topological spaces. In the example of the rugby game, a space of attacking or a space 
of defence might be enacted - depending on the relations of the various players with the ball (e.g., a relation of engagement between ball and defender who wants to keep the ball, or a relation of repulsion between the attacker who wants to kick the ball as fast as possible). Even though sociomaterial studies place less emphasis on these spatiotemporal enactments, they are equally reminiscent of topological, relational understandings, in the sense that they also argue, for instance, for a more networked understanding of objects and practices. In sum, both approaches study practices relationally, although social topology does this with a stronger focus on enacted sorts of space and time (Allen 2011; Deleuze 1994; Law 2002; Martin and Secor 2014; Murdoch 2006; Rawes 2008).

In the next paragraph, we shall shift our focus to the educational field and will, drawing on educational studies that adopt a relational and topological lens, outline how this search for forms of educational practices is effectuated, and how such research has to distinguish carefully between stating that an educational setting is constituted relationally on the one hand and showing what this constitution looks like precisely on the hand.

\subsection{Topology and educational studies}

Most educational studies drawing inspiration from social topology aim to understand educational settings in terms of forms (and consequently in terms of morphology - Masschelein and Simons 2010). Adopting the lens of homeomorphism, and aiming to compare and analyse different practices with respect to their respective form and to whether or not these forms are equivalent, seems to happen far less (Nespor 2006). The (more general) morphological lens has especially been adopted in a field designated as educational practice studies, although it is sometimes equally adopted in studies that focus on relational conceptions of space in education. Many practice studies incorporate sociomaterial and sociotopological arguments, in the sense that they attempt to give an account of how the particular form of a practice is constituted by means of a relational distribution of various actors. In a second movement, many of these studies equally stress the specific sorts of time and space and ways of doing things that are enacted (for an overview: Landri 2012). This is reminiscent of the so-called 'spatial turn' in education, which focuses on geographical conceptions of 'space' and which aims to systematically explore the various relationships between different sorts of space and educational settings (Paechter 2004; Taylor 2009). Far more than just introducing a geographical vocabulary into our conceptual understanding of education, the spatial turn is largely aimed at transcending traditional notions of scale (micro, macro) and at analysing how precisely educational spaces are relationally enacted (e.g., Beech and Larsen 2014; Larsen and Beech 2014).

Several educational studies have already adopted such a relational and topological stance. Conceived through a morphological and relational lens, for instance, McGregor (2003) accounts for typical school forms. For McGregor, what is traditionally called 'school' is not confined to the building in which relationships between various actors (pupils, teachers, maintenance team, ...) are enacted - as a traditional view would have it. In a topological conception, 'school space' is not something that would be merely 'there' by means of a couple of walls in which teachers and pupils are staying. Rather than being fixed by and to a building and hence by some extensive physical characteristics, it is precisely the other way around: it is the relations between various actors that enact school space, as a specific operation (see also Edwards and Usher 2008; Massey 1994). In a similar vein, Bayne, Gallagher, and Lamb (2014) contrast the typical form of a traditional university setting (often conceived as a delineated campus confined by the building it is housed in) with new forms that emerge by means of settings of online distance learning. They show, more specifically, how the institutional form of traditional universities is enacted by distance students, in such a way that not only bounded spatial 
understandings of the university emerge, but equally other emergent dimensions of university space as they are being enacted in these distance learning settings. Noens and Ramaekers (2014) scrutinise a typical family form, arguing that a family is not only constituted of traditional actors such as parents and their children, but that there equally are prototypical objects (e.g., a newspaper) and the relations of other family members with these objects, that enact the form of a prototypical family practice. A family, in other words, is not something that would be only qualifiable by biological (mother, father, child) or physical (house, garden, newspaper) actors, but is rather something that is constantly being done: being a family is more a matter of becoming a family, or stated otherwise: a family is constantly in the making and being relationally enacted as a family.

Heimans (2012) gives a morphological account of a typical vocational education and training (VET) form, in close connection with Australian education policy. In this account, Heimans argues that in order to scrutinise concrete implications of education policy measures, one needs to be highly attentive to how such implications take shape in practice and how the material is imbricated herein (and policy, hence, not only being a matter of discourse). Heimans continues to argue that different sorts of time and space are constantly being produced and enacted by the relations between specific education policy actors and concrete VET settings. As such, neither education policy nor VET settings are, in the first instance, characterizable as being this (policy) or that (VET) kind of setting. Rather, it is by and through the various relationships that are established between different social and material actors that specific sorts of policy and VET emerge. Similarly, Thompson and Cook (2015) unfold different topologies of contemporary educational assemblages, such as for instance an assessment topology in which global (PISA) flows are deforming the educational assemblage at local level, and analysing how these different topologies enact different sorts of space and time. Rather than making an empirically informed study, however, Thompson and Cook make an analytical argument: their focus is not so much on the empirical description of how relations between various actors enact a particular form as on the analytical postulation that the educational field is more and more characterised by global as well as local movements, mobility, change and flows. A similar argument is made by Lingard, Martino, and Rezai-Rashti (2013), who characterise educational policy movements in topological terms.

Despite the upsurge of both sociomaterial and sociotopological approaches which have become more fashionable recently, it has equally been argued that the adoption of these approaches is often limited to a mere application of a sociomaterial or sociotopological vocabulary to the educational field (Fenwick 2010; Latour 2013; Martin and Secor 2014). As these authors argue (see also Fenwick and Landri 2012), there is nevertheless a profound difference between stating that a practice is sociomaterially constituted (something that merely amounts to stating the obvious when adopting such a point of view) and presenting, first, how precisely the form of a particular setting is relationally enacted and, second, what would then be typical of this scrutinised form. The remainder of this article is devoted to these two challenges. In the following section, we argue that in order to present how an educational practice is distributed precisely - this constitutes to a large extent the central methodological challenge of studies that adopt a rigorous relational thinking - topology's emphasis on forms and figures might be beneficially adopted. For that purpose, we elucidate what a topological approach to figures and forms entails methodologically, and in relation to the descriptive texts accompanying it. In the concluding section, we emphasise the importance of equally paying attention to the typicalities of the investigated practices, drawing on Latour's $(2010,2013)$ notion of mode of existence. 


\section{Relational research}

\subsection{Data collection: scrutinising distributions}

As far as methodology is concerned, both sociomaterial and sociotopological studies are centrally concerned with finding ways of observing a setting that allows researchers to analyse the distribution of actors in this setting. As varied as these observation methods are, they are all devised in such a way that they are directed at tracing as many actors, relations between actors, and the types of actors and relations, as possible. In that sense, both sociomaterial and sociotopological approaches do not aim to be explanatory or evaluative, but concentrate in the first instance on tracing how different actors relate to/with each other (a question of data collection) in order to present a relational account of these distributions afterwards (a question of data analysis). As to data collection and well documented over the years, this tracing is largely done by ethnographic means: especially much sociomaterial research adopts an ethnographic methodology in order to understand a setting in terms of the distribution of actors and their relations in a particular practice (see Fenwick and Edwards 2010, for an overview).

As to the concrete design of such ethnographies, however, both approaches are undetermined, stating for instance that one just has to follow the actors and just has to describe - although some attempts at bringing about methodological clarification have recently been made (e.g., Latour 2005; Venturini 2010, 2012). This openness often results in studies in which the researcher starts with following one actor and expands her scope when relations between this and other actors start to unfold (e.g., Ceulemans et al. 2014; Sørensen 2009). In addition to ethnographic methods, a few other methods are adopted in educational SM and ST studies, all aiming to scrutinise the distribution of actors and relations of the setting under investigation: document (inscription) studies; interviews; analyses of digital assemblages such as websites; etc. (Decuypere, Ceulemans, and Simons 2014; Gorur 2011; Thompson 2012). Furthermore, and at the much less elaborated level of data analysis, recently many tools are being deployed with the intention of presenting and scrutinising the distributions of the settings under investigation. These tools are often visually oriented, seeking to visualise such distributions of actors and relations (Latour et al. 2012; Ruppert, Law, and Savage 2013; Savage 2009). It is to this point that we want to devote explicit attention in the next paragraph: perhaps figures are especially well-suited for presenting how (instead of merely stating that) precisely the sociomaterial distribution of educational practices takes shape.

\subsection{Data analysis: figures and texts}

With respect to the analysis and presentation of the relational distribution of different settings, we believe that such visualisations, in addition to traditional textual descriptions, might offer a fruitful contribution: figures make it possible to render the (concrete form of the) distribution of actors and relations in a particular setting intelligible. We are using the term 'figure' in a delineated manner, however, in the sense that not each usage of the term will do. More particularly, we use the term in contradistinction with terms such as 'pictures' or 'images'. Whereas pictures or images are often conceived as mimetic or representative renderings of what was to be seen in a particular setting, figures have a much more active, non-representational role: figures are descriptive objects in their own right, which, as we will argue below, make it possible to analyse a setting in terms of its form (Geerinck 2011; Savage 2009). Equally, we are not using the notion of the figure as a metaphorical device: neither are we talking about the figure as a metaphor for personality traits ('he is quite a figure'), nor as designating a particular role ('the figure of the teacher'). On the contrary, we claim that figures, as visualisations which describe something, are useful tools for analysing the distribution of 
educational settings in terms of their topological form. Conceiving of figures not as passive representations but as active devices themselves, raises some additional questions with respect to the relation of these figures with the textual body by which they are surrounded: What is the precise relation between a figure thus conceived and the text? What is the relation between the textual and the visual? Such queries are not only posing the question as to the agency that visualisations themselves have, but equally resonate with reflections about the status of 'the visible' and 'the articulable', and concomitant arguments that these two pertain to two different orders (Deleuze 1986; Foucault 1983; Savage 2009).

Deleuze, with Foucault, has argued that the visible belongs to a different realm than the articulable, and that these two can never overlap: there is no conformity between what is visible and what is articulable. Each domain, they argue, acts along its own logic and has different rules. In his studies of discourse and power, for instance, Foucault argued that what we say, that is day-to-day enunciations, is never hidden, and yet these enunciations are neither directly readable nor even sayable: each enunciation belongs to a particular regime, and such a regime supposes a delineated manner of making connections between words, sentences and propositions. The same applies to what is visible: the visible is never hidden, and yet it is neither directly seen nor visible. Rather than that, something is only rendered visible at the moment that there are devices (or machines) that shed light on some aspects, in order for something to be made visible. That is to say, Deleuze and Foucault stressed the active role that both the visible and the sayable play, and that these realms belong to two different orders. They also argued that precisely because of this non-conformity between the two domains, that is, precisely because they pertain to two different orders, intersections between these domains are sometimes established (Deleuze 1986; Foucault 1983).

It is at this point, where the visible and the articulable intersect, that what both designate as a diagram starts to emerge: a scattered combination of two domains that never overlap, that can never overlap, but where some intersection between the two orders is nevertheless being established. A diagram, then, 'is no longer an auditory or visual archive but a map, a cartography that is coextensive with the whole social field. It is an abstract machine' (Deleuze 1986, 34). It constitutes a 'meeting place' for words and images, the main quality of it not being pursuing objectivity but establishing an optical consistency by means of which the diagram is presenting a distribution of words and images in such a way that it presents the world without resembling it: the map and its markers are not the territory, the graph and its legend are not the laboratory, and so on. Rather, they are optically consistent with it: they have the capacity to look consistent with the world they depict, even though their operations are of a totally different nature (Latour 1988). A diagram (an illustrated children's book, a map with a legend, etc.) is thus a technique, or a machine, that brings the visual and the articulable together; a 'display of relations' (Deleuze 1986, 36). The diagram displays these relations in such a way that it 'never functions in order to represent a persisting world but produces a new kind of reality' (Deleuze $1986,35)$. In other words, the relations of the diagram with the world are not to be conceived in terms of passive correspondence, but rather in terms of active presentation, where that which is presented creates something new: For Foucault and Deleuze, the diagram is an active operator and furthermore the technique par excellence in which the two realms come together without ever merging.1 Hence, the diagram should not be conceived in terms of resemblance (as if the figure and the text would affirm a representation). Rather, the relations that the diagram invokes have to be conceived in terms of similitude or what Latour terms as optical consistency. 'It is no longer the finger pointing out from the canvas in order to refer to something else', Foucault says (49). The diagram does not affirm or represent anything. Rather, it presents something; offers the possibility to show 'what recognisable objects, familiar silhouettes hide, prevent from being seen, render invisible' (46). But what about the relation between the textual and the visual, then? If the diagram is not resembling the world (and vice 
versa) but standing in a relation of similitude to it, if figure and text are no longer representing an outside reality but presenting something new, how to conceive of the intersections between these two?

\subsubsection{Diagrams and forms}

To specify how to conceive of figures in a relational manner, and how these relate to the textual body by which they are surrounded, we adopt the notion of the diagram as a technique that makes it possible to display relations and that brings the visual and the textual together. In what follows, we discuss three different kinds of diagrams that not only outline the sorts of figures currently being used in sociomaterial and sociotopological studies, but equally how precisely these figures are made to stand in relation to the textual body that surrounds them.

The first is the use of sketches. In a study of how 'learning' is partly materially composed, Sørensen (2009) makes use of sketches in order to present the distribution of human (i.e., pupils and teacher) and non-human (i.e., a blackboard) actors in a classroom (Figure 1). Sørensen does not make use of such sketches with the intention of displaying/representing what really happened in the classroom that day. Rather, she deploys these sketches as templates that enable to show, that is, to present the relational distribution of an educational setting. To state that these sketches are accurate representations would thus miss the point entirely: Sørensen's intention is not to show the classroom 'as it really was', but rather to deploy sketches as descriptive renderings of particular sociomaterial aspects of the classroom that are traditionally overlooked. Furthermore, basing her argumentations on such descriptive renderings enables her to argue that this particular distribution of blackboard, teacher and pupils generates regions in a classroom (that is, a specific sort of spatiality) and enacts different sorts of presence (that is, a specific sort of temporality). In other words, a diagram of sketches and text allows Sørensen to say something about the classroom as a learning practice and eventually what is typical about that practice.

Figure 1. Sketch (Sørensen 2009, 141).

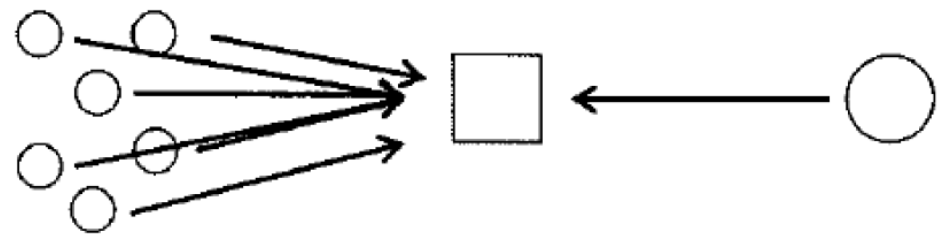

Second, recently we (Decuypere and Simons 2014a, 2014b) have made use of network visualisations (Figure 2). Especially in the field of Social Network Analysis, the use of network visualisations is getting more and more popular as a representative rendering of the social interactions that took place at a particular point in time (Knox, Savage, and Harvey 2006). This, however, is not the usage that we had in mind. Rather, what we were after is searching for ways in which network visualisations are able to present the relational distribution of several academic practices. Equally in this instance, the visualisations used are not considered as some kind of blueprint or X-ray of what contemporary academic life looks like nowadays, but precisely as topological descriptions presenting distributions of a heterogeneity of actors, or, to put it differently, as descriptions that present the particular forms of different academic practices. These (networked) forms make it possible to describe the effects that such distributions/forms generate: a network with many centres, for instance, signifies that other actors made many connections with these centres and therefore that authority was delegated to these centres. That is to say, the visualisations made it possible to present relational effects, such as for 
instance that some actors were made important in the course of one day (and not: that these were holding an a priori important position). Similar to Sørensens sketches, in this diagram of networks and text, the relation between the two realms is not that the one represents or illustrates the other. Rather, the figures allow academic practice to be rendered visible and more specifically to talk about this practice in terms of its elements, characteristics and the effects of its form in the textual descriptions surrounding the figures.

Figure 2. Network (Decuypere and Simons, 2014b, 125).

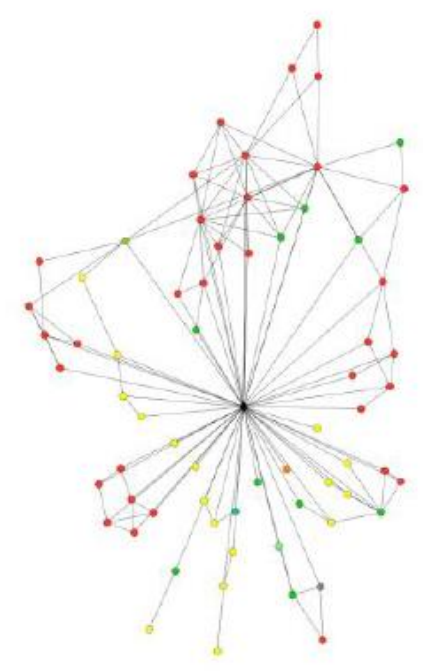

A third example is the deployment of knots as a means for topologically describing educational relations (de Freitas 2012; Figure 3). de Freitas argues for the deployment of knots as a visualisation technique that 'can function as pragmatic exercises in finding out how something works' (594, emphasis added). Such deployment of knots might assist in pointing to aspects that are traditionally more hidden from view - protest, rupture, competing forces, and so on - but are not easily read. Indeed, according to de Freitas, visualisations act as descriptive and experimental exercises, that is, not as mute renderings of reality as it is, but precisely as presentative renderings that might bring about some insight (or not). As with the two previous examples, these knots thus do not serve a representative purpose but rather try to present something in a manner that makes it challenging to read them. What do they allow us to see? How do we render them intelligible? Such questions are, according to de Freitas, no shortcomings of these visualisations but, on the contrary, an integral part of the descriptive exercise that takes place in text surrounding the diagram.

Figure 3. Knot (de Freitas 2012, 567).

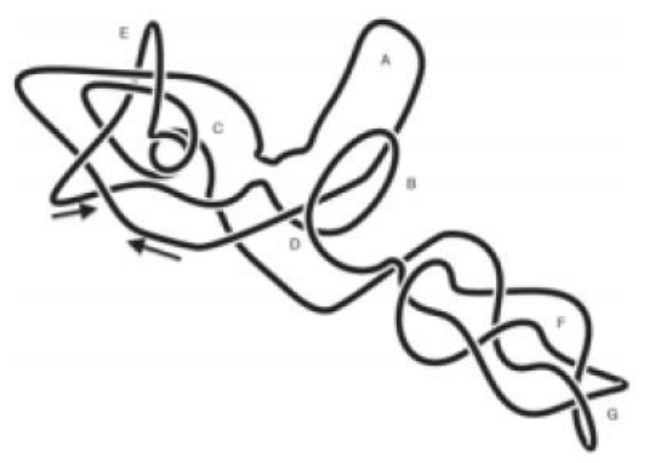


To sum up, these three examples, all three positioned in the sociomaterial and/or sociotopological field, deploy visualisations in a presentational (rather than a mimetic) and topological (rather than topographical) manner. The figures in these examples present the distribution of actors and relations (the concrete form) in a particular educational setting and serve as tools which make the relationality of different practices intelligible. As such, these figures can be conceived to be strict applications of relational thinking: they have no other aim than to present a topological distribution. Conceiving of diagrams likewise offers opportunities for scrutinising how practices are enacted, that is to say, what typicalities are present in a particular setting, which sorts of space and time such setting enacts, etc. The following section presents some techniques that might be adopted in order to compose such diagrams.

\subsubsection{Composing a diagram: ocular and writing techniques}

As argued in the previous paragraph, topologically conceived, figures present the relational distribution of an educational (i.e., classroom or academic) setting, but have no intention of faithfully resembling or representing that setting. Rather, they present how these distributions are relationally constituted and are, because of this presentational capacity, beneficial in rendering the enactments that such distributions generate (e.g., enacting different sorts of presence, actors being enacted as authorities, rupture, protest) intelligible. As such, they have to be conceived as active devices themselves that not only present a visual account of a setting to the viewer, but that equally challenge the researcher to give an adequate textual account of these forms and the effects they generate. This combination of an adequate textual and visual account was called a 'diagram'; a device by means of which the visible and the articulable intersect.

With respect to the visible, Illich (1995) conceptualised such challenges as in need of a topological training of the eye and the adoption of tailored topological ocular techniques. de Freitas, by designating knot diagrams as being exercises, hints to something similar: what these figures show is not self-evident but needs to be looked upon and reported about in a highly specific matter. That is to say, a training of the eye is needed that allows the researcher to treat such figures not in a relation of passive resemblance, but precisely in an active device relationship: what do they (enable to) show? Where to look, and looking for what precisely? It is important here to stress that the figures in a diagram act as tools of inquiry rather than as full-fledged answers. Instead of answering questions, figures more aptly raise questions with respect to the distribution of various educational settings and what this distribution then shows (Decuypere and Simons 2014b). These are challenges that need to be addressed in composing the surrounding texts. With respect to the articulable, Latour (2005; Latour, Harman, and Erdélyi 2011) conceives of such challenges as the writing of an adequate account and how this writing always necessarily entails an experimental attitude in the sense that we mentioned previously: 'writing' constitutes the laboratory of the social scientist and requires tinkering, experimentation and protocol (one might say: a training of the hands). Then again, questions arise: how to write an adequate account? What to write, and where?

Although there are no unequivocal answers to such questions, social topology can help researchers to analyse forms. Figures like the ones presented above which show the relational distribution (the form) of a certain educational setting could then be considered as ocular techniques that permit a relational gaze and allow us to look in specific ways. As such, these figures, as ocular techniques, make it possible to analyse different dimensions of different settings (a sketch is not the same as a network or a knot). Network figures, for instance, allow us to analyse boundaries (e.g., between what is included and notincluded in the network) and how such boundaries might move, stretch, expand, shrink or break 
(Hinchliffe et al. 2013; Mezzadra and Neilson 2012). In other words, figures make possible a relational gaze and train the eye topologically in the sense that they allow for raising questions such as: What do boundaries divide precisely? Are these boundaries rigid, or are there actors and/or relations that are not contained by such boundaries? This places analyses of settings at the level of how they are possibly contained in, or constrained by, certain modalities or sets of relations of a setting. Other analyses that such figures allow are analyses of regions (the upper vs. the lower half of a knot, for instance). Topologically conceived, regions point to connected and relational spaces in which different actors relate with each other.

Visualising such regions affords the possibility to relationally consider which actors relate with which others and thus to give an account of what is actually important in the conduct of a particular educational practice over and beyond singular actors: By which regions is a particular educational practice characterised? Are regions related to each other, and if so, how precisely? Are there actors that are capable of mobility between different regions (Latour et al. 2012; Law 2002)? Related hereto are analyses of settings with respect to their infrastructure, as the type of actors and relations present in a setting that allow that this setting is able to 'function'. This is for instance what sketches allow, by enacting a relational gaze that assists in pointing to the spatiotemporal effects that a blackboard generates: Which infrastructure is present in a particular educational setting? How does this infrastructure sustain this setting? (see also Mathisen and Nerland 2012). In sum, the questions raised in this paragraph are challenging ones and not easily answered, yet they are both more easily raised and answered when visualising and analysing figures that present different forms of various educational settings.

In the end, raising such questions makes it possible to say something about what precisely such settings enact. It is at this point that writing techniques come into play, techniques that allow us to give a relational account of the visible in the diagram with respect to what these figures show. How to write? Again, this question has no straightforward or unequivocal answer. In any case, this writing is not only a matter of describing what one sees, since the figures themselves are already to be conceived as descriptive objects in their own right. Rather, the central challenge of writing in a diagram amounts to composing a text with the intention of letting a practice emerge out of a particular setting: the way in which the distribution of actors and relations enacts particular ways of saying and doing things and the way in which particular sorts of time and space are enacted. More often than not, existing concepts are not always appropriate. Instead, terms that are used in a diagram need to give an account of a particular setting without trying to explain. Therefore, they often need to be tailored to the setting at hand and are therefore difficult to transpose between different studies (Decuypere and Simons 2014a; Latour 2005).

\section{Concluding: on educational modes of existence}

After having adopted such relational inquiries informed by both sociomaterial and sociotopological approaches, where does all this lead to? As we have already hinted above, it does not suffice to merely state that educational practices are sociomaterially or topologically constituted. Indeed, if one adopts a SM or ST approach, this only amounts to a generic point that can be made of each and every practice (Latour 2013). But perhaps neither does it suffice to show how an educational practice is relationally constituted. In this last section, we argue that the ultimate goal of relational thinking is to find a mode that is typical for the setting under investigation (a classroom, a higher education institution, a family, etc.). If a SM/ST view holds that it is not particularly fruitful to fix any a priori boundaries between educational and other practices or between different educational practices themselves, this is an 
analytical point of departure. In other words, neither a sociomaterial nor a sociotopological view assert that there are no differences between different practices (that might be the case, but this is never assumed a priori - neither is the contrary), but they do assert that what is typical about a particular practice is only to be found at the end of an empirical study. In that sense, the goal of SM/ST studies in education is to find words and figures which show what is typical about the educational practice under investigation (a family, school, higher education, university, ... practice) and perhaps even to define what is typical about educational practices as such. This endeavour goes way beyond a 'showing how'. Rather, we regard this as a search for the mode of existence of the practice one is studying: this might be the mode of existence of a school, a family, a university, and so on.

By the term 'educational mode of existence', we mean a particular educational way of being that makes it discernable from other (e.g., political or economic) types of being. We adopted the term from Latour's (2013) inquiry into modes of existence, which has identified fifteen different ways of being that are characteristic of 'the Moderns'. Taking stock of his entire oeuvre, Latour argues that each of these modes of being is characterised by features that are different from their counterparts. Whereas, for instance, a scientific way of existing is primarily directed at pursuing distant objectivity, a juridical way of existing is primarily directed at obtaining as much specificity as is necessary in order to be able to proceed in due course (Latour 2010, 2013).

The consequence of this is firstly that these different modes of being value different things (e.g., objectivity vs. specificity). Furthermore, and secondly, this implies that each mode of existence is identifiable by unique characteristics, and with typical ways of valuing and relating to other actors in the world. Through studying educational practices relationally, then, what becomes possible is to qualify what makes an educational practice educational and not, say, political or economical. This can only be done at the end of a study, when considering the form of a particular educational setting and the prevalent enactments that point to it being a practice.

At that point, it becomes possible to state something about these typicalities, namely which actors are necessary for a particular practice to be able to sustain itself, how these actors typically relate to each other and which type of continuity (if any) is specific to the investigated practice. Such matters refer to what was, at the beginning of this article, called homeomorphism: What are typical school forms (that is, what are typical forms of curriculum, of pedagogic approaches, of relating to the subject matters at hand, and so on)? What are typical family forms? What are typical university forms? Where are these to be found? What do they look like? Such questions pertain to (regional) ontologies in the field of education: what does it mean to exist as a school, a family, a university nowadays? In order to answer such questions, which hint at the specific mode of existence of the investigated school, family, and so on, one needs to consider the typical associations of such forms (Latour 2013; Mol 2000).

Certainly, answers to these questions will always to a certain extent be confined by the setting one has investigated. In that sense, a search for a mode of existence of the school, for instance, is not to be considered as having a generalising aim, but rather argues that there are different ways to exist as a school or university - i.e., different practices - that pertain to a similar (homeomorphic) school or university form. Yet, it is precisely in trying to answer such questions that a furthering of relational (sociomaterial and sociotopological) studies of education might lie: in offering concrete proposals as to what precisely constitutes a school mode, a family mode, a university mode and, eventually, an educational mode of existence (see also Masschelein and Simons 2013). Combining sociotopological with sociomaterial insights seems especially instructive: What is a school when approached in terms of form? Are there typical human and non-human actors, or combinations of typical actors populating a particular school? Which boundaries are solid, which more permeable in a family? Is there an infrastructure that typical academic practices share? Such are, we think, very pressing questions, 
especially in times where many educational practices are increasingly being claimed and sometimes even 'colonised' by other modes of existence, be they political, psychological or economic. The recent upsurges of conceiving of education in economical terms and consequential manifestations such as high stakes testing and rankings, or the increasing political appropriation of education are especially instructive examples in this respect (Decuypere, Simons and Masschelein 2011). If educational research (still) wants to inhabit a mode of its own, that is, being a research domain that is not usurped by other social-scientific (political, economical, etc.) ways of conceiving what education is, can, or should be, perhaps it makes sense to state that such an educational research domain and the way it exists in the broader research field, should aim to empirically present what is typical about an educational setting, and hence, by what educational practices are typically characterised. This could allow for sustaining, perhaps reinventing, a specific mode of existence of educational research itself, that would not content itself with pointing to such typicalities but that would, equally, offer propositions so as to recompose certain settings and think about what it means to re-form education.

\section{Funding}

This work was supported by the Research Foundation Flanders (FWO).

\section{Notes}

1. This is a rather loose interpretation of Deleuze's and Foucault's theorizings of the diagram. Deleuze and Foucault are centrally interested in the diagram because they consider the diagram as a central place where power is being made. Although their reflections are centrally directed at such mechanisms of power, we believe it equally to be possible to deploy the diagram in a more affirmative way and focus on what such diagrams make possible. 


\section{References}

1. Allen, J. 2011. "Topological Twists: Power's Shifting Geographies." Dialogues in Human Geography 1 (3): 283-298.10.1177/2043820611421546

2. Bayne, S., M. S. Gallagher, and J. Lamb. 2014. "Being 'at' University: The Social Topologies of Distance Students." Higher Education 67: 569-583.

3. Beech, J., and M. A. Larsen. 2014. "Replacing Old Spatial Empires of the Mind." European Education 46 (1): 75-94.10.2753/EUE1056-4934460104

4. Callon, M., and F. Muniesa. 2005. "Peripheral Vision: Economic Markets as Calculative Collective Devices." Organization Studies 26 (8): 1229-1250.10.1177/0170840605056393

5. Ceulemans, C., M. Simons, and E. Struyf. 2014. "What - if Anything - do Standards do in Education? Topological Registrations of Standardizing Work in Teacher Education." European Educational Research Journal 13 (1): 73-88.

6. De Landa, M. 2002. Intensive Science and Virtual Philosophy. London: Continuum.

7. Decuypere, M., and M. Simons. 2014a. "On the Composition of Academic Work in Digital times." European Educational Research Journal 13 (1): 89-106.10.2304/eerj

8. Decuypere, M., and M. Simons. 2014b. "An Atlas of Academic Practice in Digital times." Open Review of Educational Research 1 (1): 116-143.10.1080/23265507.2014.973899

9. Decuypere, M., C. Ceulemans, and M. Simons. 2014. "Schools in the Making: Mapping Digital Spaces of Evidence." Journal of Education Policy 29 (5): 617-639.10.1080/02680939.2013.865081

10. Decuypere, M., M. Simons, and J. Masschelein. 2011. “Perform, Measure Accurately, Optimize: On the Constitution of (evidence-based) Education Policy." International Studies in Sociologies of Education 21 (2): 115-135.

11. Deleuze, G. 1986. Foucault. Paris: Les éditions de minuit.

12. Deleuze, G. 1994. Difference and Repetition. London: Athlone Press.

13. Edwards, R. 2009. “Introduction: Life as a Learning Context?" In Rethinking Contexts for Learning and Teaching. Communities, Activities and Networks, edited by R. Edwartds, G. Biesta, and M. Thorpe, 1-13. London: Routledge.

14. Edwards, R., and R. Usher. 2008. Globalisation and Pedagogy. 2nd ed. London: Routledge.

15. Fenwick, T. 2010. "Reading Educational Reform with Actor Network Theory: Fluid Spaces, Otherings and Ambivalences." Educational Philosophy and Theory 43 (1): 114-134.

16. Fenwick, T., and R. Edwards. 2010. Actor-network Theory in Education. London: Routledge.

17. Fenwick, T., and P. Landri. 2012. "Materialities, Textures and Pedagogies." Pedagogy, Culture \& Society $20(1): 1-7$.

18. Fenwick, T., R. Edwards, and P. Sawchuk. 2011. Emerging Approaches to Educational Research: Tracing the Socio-material. London: Routledge.

19. Foucault, M. 1983. This is Not a Pipe. Translated by J. Harkness. Berkeley: University of California Press. 
20. Fox, S. 2005. "An Actor-network Critique of Community in Higher Education: Implications for Networked Learning." Studies in Higher Education 30 (1): 95-110.10.1080/0307507052000307821

21. de Freitas, E. 2012. "The Classroom as Rhizome: New Strategies for Diagramming Knotted Interactions." Qualitative Inquiry 18 (7): 557-570.10.1177/1077800412450155

22. Geerinck, I. 2011. "The Teacher as a Public Figure. Three Portraits." Unpublished doctoral diss., Leuven.

23. Gorur, R. 2011. "Policy as Assemblage." European Educational Research Journal 10 (4): 611622.10.2304/eerj

24. Habib, L., and L. Wittek. 2007. "The Portfolio as Artifact and Actor." Mind, Culture, and Activity 14 (4): 266-282.10.1080/10749030701623763

25. Heimans, S. 2012. "Coming to Matter in Practice: Enacting Education Policy." Discourse: Studies in the Cultural Politics of Education 33 (2): 313-326.

26. Hinchliffe, S., J. Allen, S. Lavau, N. Bingham, and S. Carter. 2013. "Biosecurity and the Topologies of Infected Life: From Borderlines to Borderlands." Transactions 38 (4): 531-543.

27. Illich, I. 1995. "Guarding the Eye in the Age of Show." RES: Anthropology and Aesthetics 28: 4761.

28. Knox, H., M. Savage, and P. Harvey. 2006. "Social Networks and the Study of Relations: Networks as Method, Metaphor and Form." Economy and Society 35 (1): 113140.10.1080/03085140500465899

29. Koyama, J. P., and H. Varenne. 2012. "Assembling and Dissembling: Policy as Productive Play." Educational Researcher 41 (5): 157-162.10.3102/0013189X12442799

30. Landri, P. 2012. "A Return to Practice: Practice-Based Studies of Education." In Practice, Learning and Change, edited by P. Hager, A. Lee, and A. Reich, 85-100. Dordrecht: Springer.

31. Landri, P., and E. Neumann. 2014. "Introduction. Mobile Sociologies of Education." European Educational Research Journal 13 (1): 1-8.10.2304/eerj

32. Larsen, M. A., and J. Beech. 2014. "Spatial Theorizing in Comparative and International Education Research." Comparative Education Review 58 (2): 191-214.10.1086/675499

33. Lash, S. 2012. "Deforming the Figure: Topology and the Social Imaginary." Theory, Culture \& Society 29 (4-5): 261-287.

34. Latour, B. 1988. "Visualization and Cognition. Drawing Things Together." Knowledge and Society 6: $1-40$.

35. Latour, B. 2005. Reassembling the Social. Oxford: Oxford University Press.

36. Latour, B. 2010. The Making of Law. Cambridge: Polity Press.

37. Latour, B. 2013. An Inquiry into Modes of Existence. Cambridge, MA: Harvard University Press.

38. Latour, B., G. Harman, and P. Erdélyi. 2011. The Prince and the Wolf. Winchester: Zero Books. 
39. Latour, B., P. Jensen, T. Venturini, S. Grauwin, and D. Boullier. 2012. "'The Whole is Always Smaller than Its Parts' - A Digital Test of Gabriel Tarde's Monads." The British Journal of Sociology 63 (4): 590-615.10.1111/bjos.2012.63.issue-4

40. Law, J. 2002. "Objects and Spaces." Theory, Culture \& Society 19 (5/6): 91-105.

41. Law, J. 2009. "Actor Network Theory and Material Semiotics." In The New Blackwell Companion to Social Theory, edited by Bryan S. Turner, 141-158. Malden, MA:

Blackwell.10.1002/9781444304992.ch7

42. Lingard, B., W. Martino, and G. Rezai-Rashti. 2013. "Testing Regimes, Accountabilities and Education Policy: Commensurate Global and National Developments." Journal of Education Policy 28 (5): 539-556.10.1080/02680939.2013.820042

43. Luck, J. T. 2008. Lost in Translations: A Socio-technical Study of Interactive Videoconferencing at an Australian University. http://p8080-

acquire.cqu.edu.au.kuleuven.ezproxy.kuleuven.be/vital/access/manager/Repository/cqu:4525

44. Lury, C., L. Parisi, and T. Terranova. 2012. "Introduction: The Becoming Topological of Culture." Theory, Culture \& Society 29 (4-5): 3-35.

45. Marres, N. 2012. "On Some Uses and Abuses of Topology in the Social Analysis of Technology (or the Problem with Smart Meters)." Theory, Culture \& Society 29 (4-5): 288-310.

46. Martin, L., and A. J. Secor. 2014. "Towards a Post-mathematical Topology." Progress in Human Geography 38 (3): 420-438.10.1177/0309132513508209

47. Masschelein, J., and M. Simons. 2010. Jenseits der Exzellenz: Eine kleine Morphologie der WeltUniversität [Beyond Excellence: A Small Morphology of the World University]. Regensburg:

Diaphanes.

48. Masschelein, J., and M. Simons. 2013. In Defense of the School. Leuven: E-ducation, Culture \& Society.

49. Massey, D. 1994. Space, Place and Gender. Cambridge: Polity Press.

50. Mathisen, A., and M. Nerland. 2012. "The Pedagogy of Complex Work Support Systems: Infrastructuring Practices and the Production of Critical Awareness in Risk Auditing." Pedagogy, Culture \& Society 20 (1): 71-91.

51. McGregor, J. 2003. "Making Spaces: Teacher Workplace Topologies." Pedagogy, Culture \& Society 11 (3): 353-377.

52. Mezzadra, S., and B. Neilson. 2012. "Between Inclusion and Exclusion: On the Topology of Global Space and Borders." Theory, Culture \& Society 29 (4-5): 58-75.

53. Mol, A. 2000. "Dit is geen programma. over empirische filosofie [This is not a Program. On Empirical Philosophy]." Krisis 1 (1): 6-26.

54. Mol, A., and J. Law. 1994. "Regions, Networks and Fluids: Anaemia and Social Topology." Social Studies of Science 24 (4): 641-671.10.1177/030631279402400402

55. Murdoch, J. 2006. Post-structuralist Geography: A Guide to Relational Space. London: Sage.

56. Nespor, J. 2004. “Educational Scale-making." Pedagogy, Culture and Society 12 (3): 309326.10.1080/14681360400200205 
57. Nespor, J. 2006. "Morphologies of Inquiry: The Uses and Spaces of Paradigm Proliferation 1." International Journal of Qualitative Studies in Education 19 (1): 115128.10.1080/09518390500450268

58. Noens, P., and S. Ramaekers. 2014. "The Family as a 'Gathering': How the Life of an Object 'Makes' a Family." International Journal of Child, Youth and Family Studies 4.2 (5): 722-740.

59. Orlikowski, W. J. 2007. "Sociomaterial Practices: Exploring Technology at Work." Organization Studies 28 (9): 1435-1448.10.1177/0170840607081138

60. Paechter, C. 2004. "Space, Identity and Education." Pedagogy, Culture and Society 12 (3): 307308.10.1080/14681360400200204

61. Plotnisky, A. 2003. "Algebras, Geometries and Topologies of the Fold." In Between Deleuze and Derrida, edited by P. Patton and J. Protevi, 98-119. New York: Continuum.

62. Rawes, P. 2008. Space, Geometry and Aesthetics. Palgrave Macmillan.10.1057/9780230583610

63. Ruppert, E., J. Law, and M. Savage. 2013. "Reassembling Social Science Methods: The Challenge of Digital Devices." Theory, Culture \& Society 30 (4): 22-46.

64. Savage, M. 2009. "Contemporary Sociology and the Challenge of Descriptive Assemblage." European Journal of Social Theory 12 (1): 155-174.10.1177/1368431008099650

65. Schatzki, T. R. 2010. “Materiality and Social Life." Nature and Culture 5: 123-149.

66. Serres, M. 1979. Le passage du Nord-Ouest [The Northwest Passage]. Paris: Editions de Minuit.

67. Serres, M., and B. Latour. 1995. Conversations on Science, Culture, and Time. Ann Arbor, MI: University of Michigan Press.

68. Sha, X. W. 2012. “Topology and Morphogenesis." Theory, Culture, \& Society 19 (4-5): 220-246.

69. Sørensen, E. 2009. The Materiality of Learning. Cambridge, MA: Cambridge University Press.10.1017/СBO9780511576362

70. Taylor, C. 2009. "Towards a Geography of Education." Oxford Review of Education 35 (5): 651669.10.1080/03054980903216358

71. Thompson, T. L. 2012. “I'm Deleting as Fast as I Can: Negotiating Learning Practices in Cyberspace." Pedagogy, Culture \& Society 20 (1): 93-112.

72. Thompson, G., and I. Cook. 2015. "Becoming-topologies of Education: Deformations, Networks and the Database Effect." Discourse: Studies in the Cultural Politics of Education 5 (36): 732-748.

73. Venturini, T. 2010. "Diving in Magma: How to Explore Controversies with Actor-network Theory." Public Understanding of Science 19 (3): 258-273.10.1177/0963662509102694

74. Venturini, T. 2012. "Building on Faults: How to Represent Controversies with Digital Methods." Public Understanding of Science 21 (7): 796-812.10.1177/0963662510387558

75. Waltz, S. B. 2006. "Nonhumans Unbound: Actor-network Theory and the Reconsideration of 'Things' in Educational Foundations." Educational Foundations 20: 51-68. 